\title{
Characterization of the BnA10.tfl1 Gene Controls Determinate Inflorescence Trait in Brassica napus L.
}

\author{
Yongpeng Jia $1,2,3,4,5,+$, Kaixiang Li ${ }^{1,2,3,4,5,+}$, Haidong Liu $1,2,3,4,5$, Lingxiong Zan $1,2,3,4,5$ and \\ Dezhi Du $1,2,3,4,5, *$
}

1 State Key Laboratory of Plateau Ecology and Agriculture of Qinghai University, Academy of Agricultural and Forestry Sciences, Qinghai University, Xining 810016, Qinghai, China; ypjia2018@163.com (Y.J.); 18997174190@163.com (K.L.); dahaima@163.com (H.L.); 15003619373@163.com (L.Z.)

2 Key Laboratory of Spring Rapeseed Genetic Improvement of Qinghai Province, Xining 810016, Qinghai, China

3 The Qinghai Research Branch of the National Rapeseed Genetic Improvement Center, Xining 810016, Qinghai, China

4 Spring and Rape Scientific Observation Experimental Station of the Ministry of Agriculture and Rural Areas, Xining 810016, Qinghai, China

5 Qinghai Research and Development Center for Spring Rapeseed, Xining 810016, Qinghai, China

* Correspondence: qhurape@126.com; Tel.: +86-0971-5366-520

+ The authors contributed equally to this article.

Received: 12 September 2019; Accepted: 5 November 2019; Published: 7 November 2019

\begin{abstract}
Determinate inflorescences have a significant effect on the genetic improvement of rapeseed, so understanding the molecular function underlying the inflorescence trait may be beneficial to oilseed breeding. A previous study found candidate gene BnTFL1 (Terminal Flower 1) for control of the inflorescence trait on Brassica napus chromosome A10 (16,627-16,847 kb). However, little is known about the function of the BnTFL1 gene in B. napus. In this study, we firstly studied the formation of the shoot apical meristem and gene expression in indeterminate and determinate inflorescences; the results showed that the inflorescence architecture and BnA10.TFL1 expression showed significant differences in the shoot apex at the budding stage. Then, two alleles (named BnA10.TFL1 gene from indeterminate and $B n A 10 . t f l 1$ gene from determinate) were cloned and sequence-analyzed; the results suggest that the open reading frame of the alleles comprises $537 \mathrm{bp}$, encodes 178 amino acids containing a conserved phosphatidylethanolamine-binding protein (PEBP) domain, and shares high similarity with Arabidopsis thaliana TFL1. To analyze the function of BnA10.TFL1, the BnA10.TFL1 gene was introduced into the determinate $A$. thaliana $f f l$ mutant and B. napus 571 line by complementation experiment. The determinate traits were restored to indeterminate, and expression of BnA10.TFL1 was increased in the indeterminate shoot apex. These results reveal that BnA10.tfl1 is a gene controlling the determinate inflorescence trait. Moreover, the BnA10.TFL1 protein was localized to the nucleus, cytoplasm, and plasma membrane. Collectively, the results of this study help us to understand the molecular mechanism of determinate inflorescences and will provide a reliable research basis for the application of determinate inflorescences in B. napus.
\end{abstract}

Keywords: Brassica napus; Terminal Flower 1; indeterminate; determinate; transformation; quantitative real-time PCR

\section{Introduction}

Rapeseed is a very important oil crop around the world. Brassica napus has the largest planting area among the three rapeseed cultivar species (B. napus, AACC, $2 n=38$; Brassica juncea, AABB, $2 n=36$; Brassica rapa, AA, $2 n=20$ ) in China [1,2]. The wild type of $B$. napus has an indeterminate 
inflorescence. In production, the indeterminate growth habits have shown some defects, such as higher plants, easier lodging, long growth periods, and inconsistent ripening periods. With the realization of crop mechanization, overcoming the deficiency of indeterminate growth habits is a problem that breeders must face. However, it provides an opportunity for breeders to realize a transformation from indeterminate inflorescences to determinate inflorescences in plants. Current studies have shown that the determinate inflorescence mutant in B. napus has the characteristics of reduced plant height, lodging resistance, early growth period, and no negative impact on yield, which can complement the shortcomings of the indeterminate inflorescence growth habits in B. napus [3]. Therefore, it is essential to research the molecular mechanism of determinate inflorescences for the genetic improvement of B. napus.

At present, there are many reports on determinate inflorescence traits in plants [4-7]. The determinate inflorescence mutant $t f l 1$ gene was screened from wild-type Arabidopsis thaliana using treatment ethylmethans sulfonate (EMS). The $t f l 1$ mutant shoot apex produces a terminal flower, preventing further shoot apical meristem (SAM) differentiation, and determinate growth of $t f l 1$ can reduce plant height and early flowering. In contrast, the inflorescence SAM of wild-type A. thaliana, which has indeterminate growth, retains its differentiation capacity and continues to generate new buds; thus, it increases the plant height and growth period $[8,9]$. In leguminous crops, determinate inflorescence plants, controlled by Vrdet1 (TFL1 homologous), had earlier ripening pods than indeterminate plants [10]. B. juncea commercial varieties showed that determinate inflorescence plants had superior traits (plant height, flowering time, maturity consistency, harvest index, 1000-grain weight, etc.) when compared to indeterminate inflorescence plants [6]. In B. napus, Li et al. [3] assessed indeterminate and determinate plants from the $\mathrm{BC} 3$ population; the results showed that the plant height, maturation period, and number of branches in determinate inflorescence plants were more advantageous than those in the indeterminate plants. Thus, further study of the determinate trait gene has important guiding significance for crop breeding.

The SAM signaling pathways and inflorescence architecture have also been studied in A. thaliana [11,12]. The inflorescence architecture gene TFL1 interacts with Flowering Locus $D(F D)$ to affect the expression levels of $L E A F Y(L F Y)$. TFL1 also regulates the expression of downstream genes APETALA1 (AP1) and CAULIFLOWER (CAL). Intriguingly, LFY and AP1 may affect normal expression of the TFL1 gene in the SAM; thus, indeterminate inflorescences may continuously produce flowers $[13,14]$. These genes ( $L F Y, A P 1$, and $C A L$ ) share major functions in floral meristem identity [15,16]. Meanwhile, studies have consistently indicated that $A P 1$, a MADS-box transcription factor, is also a floral organ identity gene that acts in the development of floral sepals and petals; among the other floral organ identity genes, APETALA3 (AP3) and PISTILLATA (PI) may control the development of petals and stamens [17-19]. In cucumber, CsTFL1 interacts with CSNOT2a to inhibit determinate inflorescences and terminal flower formation, but low expression of CsTFL1 caused non-interaction with CSNOT2a to produce determinate growth [20]. In Phaseolus vulgaris, PvTFL1y restored normal indeterminate growth, similar to that of wild-type A. thaliana, when it was introduced into the $f f l 1$ mutant, which has determinate inflorescences [21]. Therefore, the $t f l 1$ gene is essential for the maintenance and control of determinate plant growth.

Recent research has reported the determinate inflorescence trait in $B$. napus, and genetic studies showed that a related candidate gene, BnTFL1 on chromosome A10, is homologous to the A. thaliana TFL1 [3]. However, little is known about the function of TFL1 in B. napus. In the present study, we mainly identified the function of BnA10.TFL1 from indeterminate inflorescence plants by the complementation test. The full-length sequence containing the BnA10.TFL1 gene was introduced into the determinate $A$. thaliana $t f l 1$ mutant and the determinate B. napus 571 line. The results indicated that the BnA10.tfl1 gene may control the determinate inflorescence trait in B. napus. 


\section{Materials and Methods}

\subsection{Plant Materials}

In this study, two B. napus lines, the indeterminate inflorescence line 2014 and the determinate inflorescence line 4769, were used as materials. Using 2014 as the donor parent and 4769 as the recurrent parent, near-isogenic lines (NIL) were constructed after three consecutive selective backcrosses and selfing for two generations. These offspring were then divided into homozygous groups for indeterminate and determinate NIL. These plants were grown in an experimental field at Qinghai University, Xining, China. All required routine field management was carried out.

An early maturing $B$. napus line with determinate inflorescences, named 571 (carrying the 4769 allele at the BnA10.tfl1 locus), was used as the transformation recipient. We also purchased the $A$. thaliana CS3091 mutant ( $t f l 1-2$, determinate inflorescences) from the Arabidopsis Biological Resource Center (ABRC). Transgenic plants were grown in growth chambers $(10,000 \mathrm{Lux})$ under $16 \mathrm{~h}$ light at $24{ }^{\circ} \mathrm{C}$ and $8 \mathrm{~h}$ dark at $16^{\circ} \mathrm{C}$. The total DNA of the plants was isolated using the CTAB (cetyltrimethylammonium bromide) method [22,23].

\subsection{Paraffin Sectioning and Scanning Electron Microscopy Analysis}

Previous studies have found that the determinate phenotype was caused by the development and differentiation of the shoot apical meristem (SAM) [3-5,14], To further understand the phenotypic differences of apical SAM, we observed the inflorescence SAM in homozygous indeterminate and determinate plants at different stage. Paraffin sectioning of the plant SAMs was performed as previously described [24]. The samples were treated with 50\% FAA (Formalin-acetic acid-alcohol), then safranine-dyed, rinsed, dehydrated, cleared, infiltrated, embedded, sliced, sealed, and examined with a Nikon microscope (Nikon, Tokyo, Japan) [25]. Scanning electron microscopy (SEM) was performed following the previously described methods [26]: the SAMs were transferred to $2.5 \%$ glutaraldehyde and then rinsed, fixed again, and dehydrated. After drying and spraying with gold powder, they were imaged using a JSM-6610LV scanning electron microscope (JEOL Ltd., Tokyo, Japan).

\subsection{Gene Cloning and Sequencing}

Sequence of the gene was obtained in the BRAD (http://brassicadb.org/brad/) based on the homologous to TFL1 from A. thaliana (AT5G03840). Specific primers (TFL1-orf-F/TFL1-orf-R) were designed based on this sequence (Table S1). The gDNA and cDNA of BnA10.TFL1/BnA10.tfl1 were isolated from indeterminate and determinate plants, and the sequences were cloned using PMD18-T vector and E. coli DH5a methods. The six positive clones which were verified with M13-specific primers were sequenced by Sangon Biotech (Shanghai, China). To obtain proteins homologous to BnA10.TFL1 from other species, the BnA10.TFL1 amino acid sequence was deduced and downloaded using GeneDoc (http://www.nrbsc.org/gfx/genedoc/) and NCBI (https://blast.ncbi.nlm.nih.gov/) BlastP. The phylogenetic tree was analyzed using the neighbor-joining method and 1000 bootstrap replicates in MEGA 7.0 (https://www.megasoftware.net/). The homologous proteins in NCBI were obtained, including BrTFL1 (XP_009122666.1) in B. rapa, BcTFL1 (ATQ37959.1) in B. carinata, BjTFL1 (ATQ37953.1) in B. juncea, BoTFL1 (BAA33420.1) in B. oleracea, AtTFL1 (AAM27947.1) in A. thaliana, RsTFL1 (XP_018474870.1) in Raphanus satious, ThTFL1 (XP_010543489.1) in Tarenaya hassleriana, CcTFL1 (XP_006430226.1) in Citrus clementina, VcTFL1 (ART91289.1) in Vaccinium corymbosum, RiTFL1 (AXF50634.1) in Rubus idaeus, GmTFL1 (ACJ61501.1) in Glycine max, LaTFL1 (XP_019444254.1) in Lupinus angustifolius, DlTFL1 (AGT41971.1) in Dimocarpus longan, FaTFL1 (AMR34693.1) in Fragaria $\times$ ananassa, VuTFL1 (AIA10351.1) in Vigna unguiculata, HbTFL1 (XP_021639026.1) in Hevea brasiliensis, and GsTFL1 (KHN18765.1) in Glycine soja. 


\subsection{Plasmid Construction}

We explored the function of BnA10.TFL1 via complementation tests. The primers were designed with NCBI Primer-BLAST (https://www.ncbi.nlm.nih.gov/tools/primer-blast/) using the BnaA10g26300D (TFL1 homologous) sequences from the BRAD. The enzyme restriction sites EcoRI and PstI and $15 \mathrm{bp}$ sequences from both sides of the pCAMBIA2300 vector were added to the ends of the primers (Table S1). The fragment of genomic DNA that included the upstream, full-length gene, and downstream sequences was amplified using Phusion Hot Start High Fidelity DNA polymerase (NEB, Ipswich, MA, USA) with recombinant primers (CP525-F/CP525-R, Table S1) from the indeterminate inflorescence line 2014. The complementation plasmid $p B n A 10 . T F L 1: B n A 10 . T F L 1$ was produced using a sequenced genomic fragment and the pCAMBIA2300 vector that had been digested with EcoRI and PstI using a One Step Cloning Kit (Vazyme, Nanjing, China), as previously described [24]. The confirmed recombinant plasmid was transformed into GV3101 (Agrobacterium tumefaciens) and stored at $-80^{\circ} \mathrm{C}$ after adding sterilized glycerin.

\subsection{Plant Transformation}

For transformation of $A$. thaliana, we used the floral dip method described previously [27]. The fused construct was introduced into the A. thaliana tfl1-2 mutant. For transformation of B. napus, the complementation plasmid, $p B n A 10 . T F L 1: B n A 10 . T F L 1$, was transformed into the determinate inflorescence 571 through the Agrobacterium-tumefaciens-mediated hypocotyl method [28]. The positive transgenic plants were confirmed and identified by antibiotic selection and PCR (Table S1).

\subsection{RNA Extraction and Quantitative Real-time PCR}

Total RNA was isolated from the plants using a TaKaRa MiniBEST Universal RNA Extraction Kit (TaKaRa, Dalian, China), while cDNA synthesis was performed using a PrimeScriptTM RT Reagent Kit with gDNA Eraser (Perfect Real Time) (TaKaRa, Dalian, China). The primers for qRT-PCR were designed with Primer-BLAST in NCBI (https://www.ncbi.nlm.nih.gov/tools/primer-blast/). For the relative quantification of transcriptional levels, Actin7 was used as the reference gene (Table S1), as recommended by ICG (http://icg.big.ac.cn/index.php/Brassica_napus) [29]. qRT-PCR was performed on a LightCycler ${ }^{\circledR} 480$ Instrument II (Roche, Basel, Switzerland) in a total volume of $20 \mu \mathrm{L}$ containing $2 \mu \mathrm{L}$ gene-specific primers $(10 \mathrm{ng} / \mu \mathrm{L}), 8 \mu \mathrm{L}$ cDNA $(12.5 \mathrm{ng} / \mu \mathrm{L})$, and $10 \mu \mathrm{L}$ SYBR ${ }^{\circledR}$ Premix Ex Taq ${ }^{\mathrm{TM}}$ (Tli RNaseH Plus) (TaKaRa, Dalian, China). qRT-PCR was performed in three biological replicates and three technical replicates. The data were processed using the $2^{-\triangle \Delta C T}$ method [30].

\subsection{Subcellular Localization of the BnA10.TFL1 Protein}

To analyze the subcellular localization of the BnA10.TFL1 protein, primers (without the stop codon "TAA") relating to subcellular localization were designed with Primer5 using the cDNA sequence. Then, the enzyme restriction sites $\mathrm{XbaI}$ and $\mathrm{BamHI}$ and the homologous sequences from the pCAMBIA3301-GFP vector were added to the ends of the primer. The cDNA of BnA10.TFL1 was amplified using Phusion Hot Start High Fidelity DNA polymerase (NEB, Ipswich, MA, USA) with recombinant primers (XWB-3301-XbaIF/XWB-3301-BamHIR, Table S1) from the indeterminate inflorescence line 2014. The confirmed sequence was inserted into the modified pCAMBIA3301-GFP vector (containing the GFP gene) that had been digested by XbaI and BamHI using a One Step Cloning Kit (Vazyme, Nanjing, China). The fusion plasmid BnA10.TFL1:GFP, transformed into GV3101 (Agrobacterium tumefaciens), was introduced into the leaf epidermal cells of Nicotiana benthamiana by injection methods, as previously described [31]. The GFP signals were obtained using a confocal laser scanning microscope (Leica, Wetzlar, Germany). 


\section{Results}

\subsection{Observations of the SAM Apex in B. napus}

To find the developmental stage at which SAM architecture variation originates in the determinate trait, we observed the formation of the SAM in indeterminate and determinate plants by scanning electron microscopy and paraffin sectioning techniques (Figure 1). The SAM arquitecture showed no differences between the indeterminate (Figure $1 \mathrm{a}, \mathrm{b}, \mathrm{g}, \mathrm{h}$ ) and determinate (Figure 1d,e,j,k) plants during both the vegetative meristem (two leaves) and early inflorescence meristem stages (four leaves). At the budding stage (eight leaves), we observed that SAM of indeterminate inflorescence (Figure 1c,i) was similar to that of two and four leaves stages. However SAM of the determinate inflorescence (Figure 1f,l) showed morphology similar to the floral organ. These findings can help us to provide a reference for the observation and sampling of determinate inflorescences in $B$. napus.

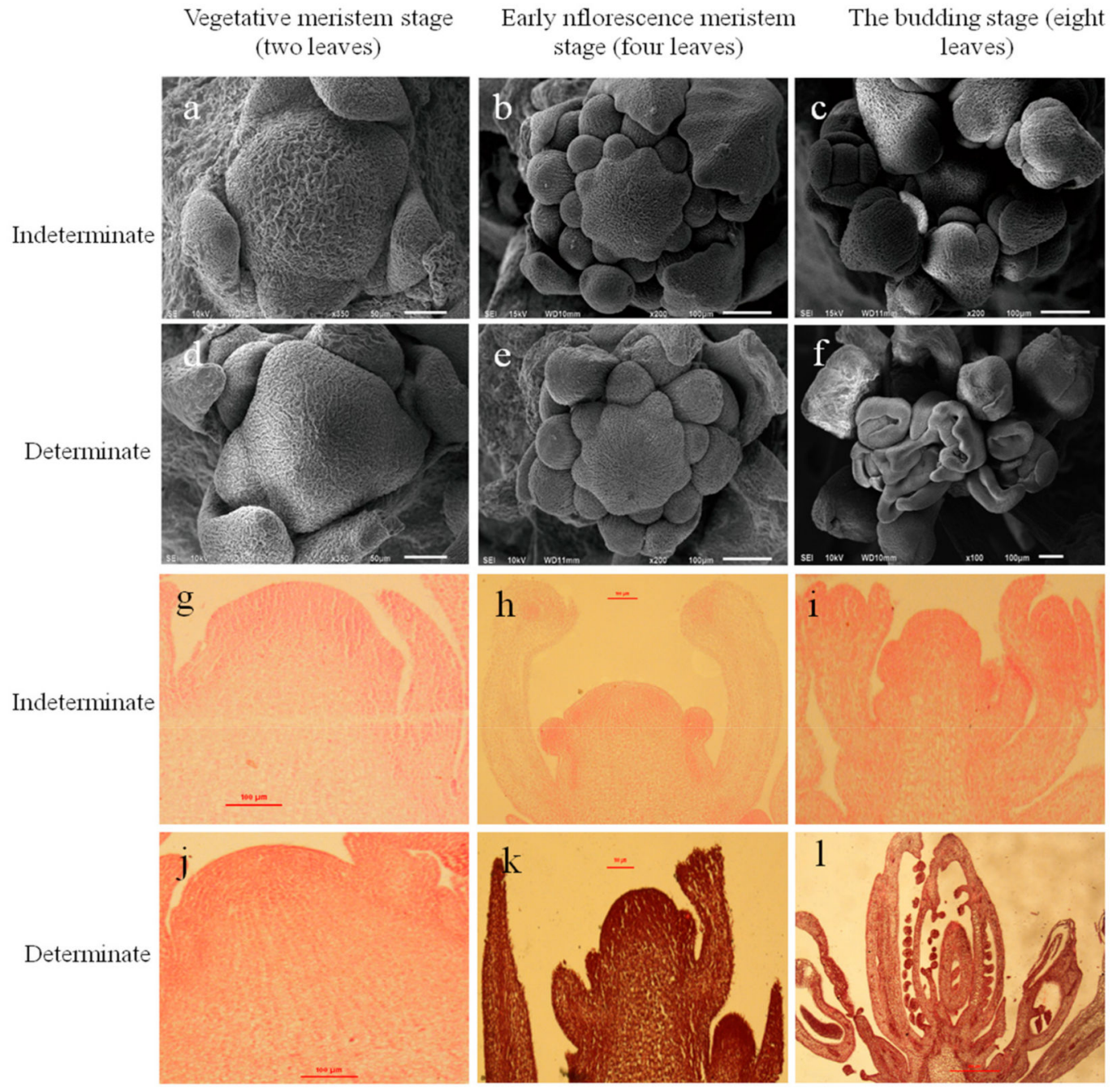

Figure 1. Scanning electron microscopy and paraffin section images of indeterminate (a-c,g-i) and determinate $(\mathbf{d}-\mathbf{f}, \mathbf{j}-\mathbf{l})$ shoot apical meristems (SAMs). (a,d, $\mathbf{g}, \mathbf{j})$ SAMs of indeterminate and determinate inflorescences at the vegetative growth stage (two leaves). (b,e,h, $\mathbf{k})$ SAMs of indeterminate and determinate inflorescences at the early inflorescence meristem stage (four leaves). (c,f,i,il) SAMs of indeterminate and determinate inflorescences during the budding period (eight leaves). 


\subsection{Expression of BnA10.TFL1 in Different Tissues of B. napus}

The expression levels of BnA10.TFL1 were examined using qRT-PCR in different tissues between the indeterminate and determinate NIL, including the shoot apex at the budding stage, roots, stems, leaves, and flowers (Figure 2). The expression levels of BnA10.TFL1 in the shoot apex were higher than those in the other tissues. However, BnA10.TFL1 was significantly up-regulated in the shoot apex of the indeterminate NIL compared with the determinate NIL at the budding stage (Figure $2,{ }^{* *} p<0.01$ ). The results indicated that BnA10.TFL1 may be specifically expressed in the shoot apex. This is similar to the expression of the TFL1 gene in A. thaliana. Thus, the expression levels of BnA10.TFL1 may cause changes in the determinate inflorescence trait.

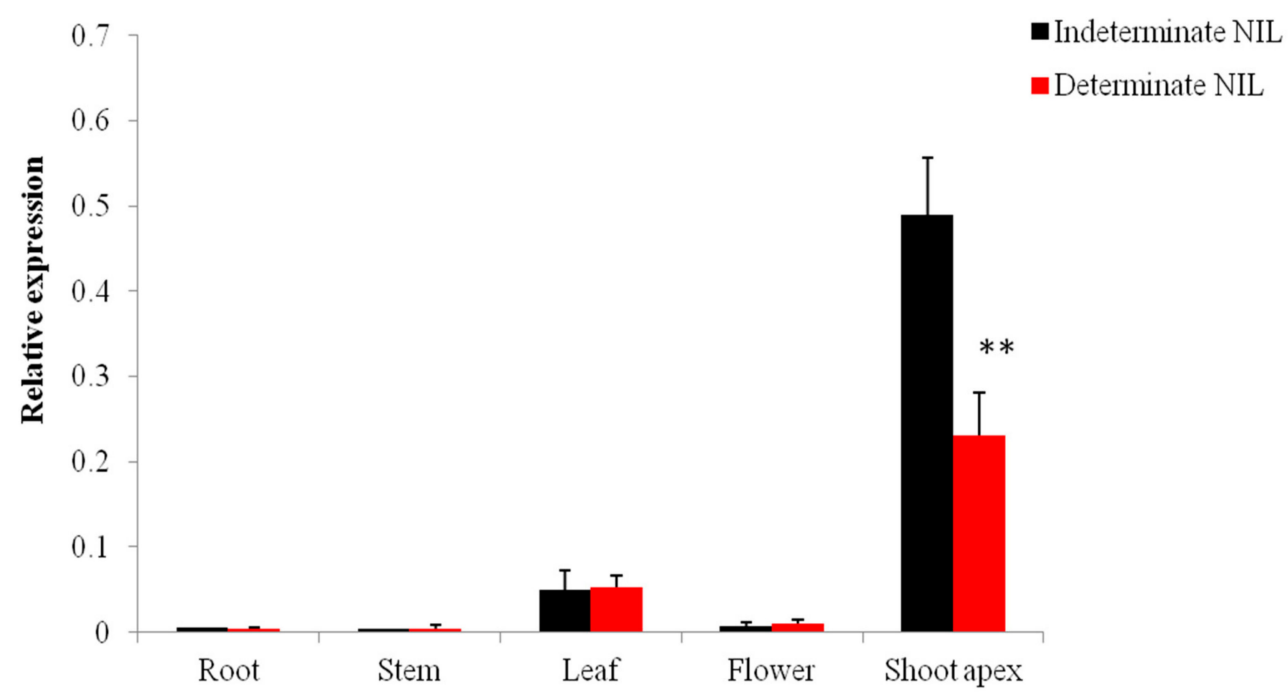

Figure 2. Expression of the BnA10.TFL1 gene in different tissues of indeterminate and determinate near-isogenic lines (NIL) in B. napus. The error bars show the standard errors from three biological replicates. ${ }^{* *}$ indicates significant differences at $p<0.01$.

\subsection{Cloning and Sequencing Analysis of the BnA10.TFL1/BnA10.tfl1 Genes}

A previous study reported that BnTFL1, being homologous to the TFL1 gene of $A$. thaliana, is a candidate gene for the inflorescence trait in B. napus chromosome A10 [3]. This candidate gene was isolated from indeterminate and determinate plants, and the two alleles were named BnA10.TFL1 and BnA10.tfl1. The sequences of the BnA10.TFL1/BnA10.tfl1 gene were cloned from the indeterminate line 2014 and the determinate lines 4769/571 using the specific primer (TFL1-orf-F/TFL1-orf-R) by PCR amplification (Table S1). In 2014 (indeterminate) (Figure 3a), 1066 bp gDNA and $537 \mathrm{bp}$ cDNA fragment sequences of BnA10.TFL1 were found, and these included four exons and three introns by Blastn analysis. In 4769/571 (determinate) (Figure 3a), the sequences of BnA10.tfl1 also had four exons and three introns; the gDNA and full-length cDNA of the gene comprised $1072 \mathrm{bp}$ and $537 \mathrm{bp}$, respectively. Sequence analysis of the gDNA and cDNA of both BnA10.TFL1 and BnA10.tfl1 revealed that there are differences between 2014 (indeterminate) and 4769/571 (determinate) (Figure 3a): there are four single-nucleotide polymorphism (SNP) mutations in the exons and $18 \mathrm{SNP} /$ Indel in the introns (15 SNP mutations, two insertions of $6 \mathrm{bp}$ and $2 \mathrm{bp}$, and one instance of $2 \mathrm{bp}$ deletion).

The amino acids were analyzed using GeneDoc and NCBI BlastP. The results indicated that the BnA10.TFL1 and BnA10.tfl1 alleles encode 178 amino acids; only two different amino acids were observed i.e., the phenylalanine (F) and leucine (L) in indeterminate 2014 becomes the leucine (L) and phenylalanine (F) in determinate 4769 (571) (Figure 3b). In addition, this protein contains a conserved phosphatidylethanolamine-binding protein (PEBP) domain and belongs to the PEBP family, and the amino acid sequence of BnA10.TFL1 showed 85\% identity to $A$. thaliana TFL1 (AT5G03840) (Figure 3b). The phylogenetic analysis showed that the BnA10.TFL1 and TFL1 proteins from other plant species 
can be divided into two separate clades; BnA10.TFL1 was grouped closely with AtTFL1 (AAM27947.1) from A. thaliana (Figure 3c).

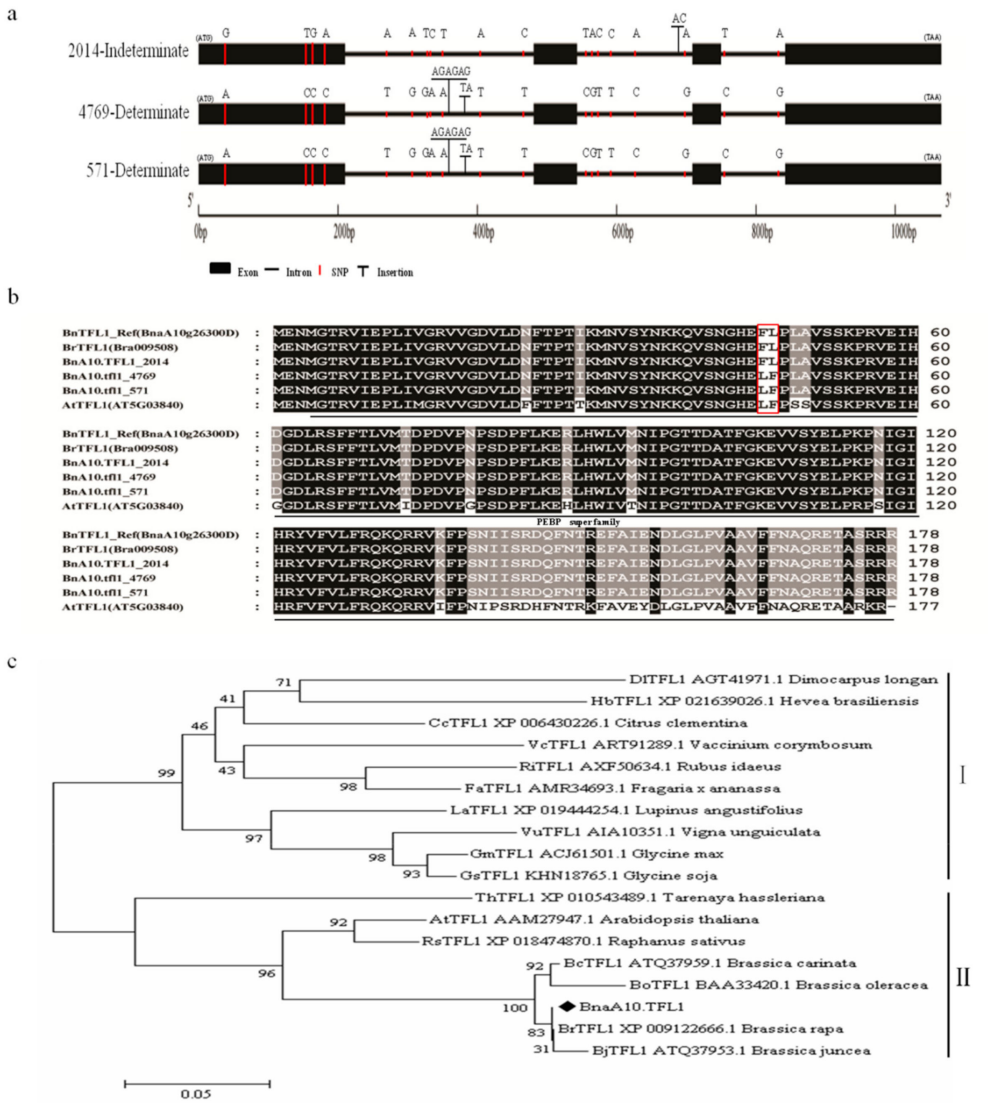

Figure 3. Sequence analysis of BnA10.TFL1. (a) Gene structure and natural variations of the alleles from the indeterminate line 2014 and determinate lines 4769/571 of BnA10.TFL1/BnA10.tfl1. (b) Amino acid sequences of BnA10.TFL1 and BnA10.tfl1 from the indeterminate 2014 and determinate 4769/571 plants were aligned with those of other species, including A. thaliana AtTFL1 (AT5G03840), BnTFL1_Ref (BnaA10g26300D) from the B. napus reference genome, and BrTFL1 (Bra009508) from the B. rapa reference genome. The black line indicates the Phosphatidylethanolamine-binding protein (PEBP) domain. The red border indicates the position of amino acid difference between indeterminate 2014 and determinate 4769 (571). (c) Phylogenetic tree of TFL1 homologous proteins. These proteins are marked with their gene names and NCBI accession numbers. The black square indicates BnA10.TFL1, I and II show the two separate clades.

\subsection{BnA10.TFL1 Rescues the tfl1-2 Mutant Phenotype in A. thaliana}

To confirm the function of BnA10.TFL1 in A. thaliana, the full-length genomic DNA of BnA10.TFL1 amplified from the indeterminate line 2014 was used to construct pBnA10.TFL1:BnA10.TFL1 in the pCAMBIA2300 vector. This vector, containing a $3816 \mathrm{bp}$ genomic fragment incorporating the BnA10.TFL1 gene (1860 bp upstream, $1066 \mathrm{bp}$ coding region, and $890 \mathrm{bp}$ downstream) (Figure S1) was transformed into the $A$. thaliana tfl1-2 mutant (determinate inflorescences). The transgenic $A$. thaliana and $t f l 1-2$ mutants showed the expected results (Figure $4 \mathrm{~b}-\mathrm{d}$ ). The transgenic lines (Figure $4 \mathrm{c}, \mathrm{d}$ ) had indeterminate inflorescence stem growth similar to that of wild-type $A$. thaliana (Figure 4a). The terminal flowers of $t f l 1-2$ mutants (Figure $4 \mathrm{~b}$ ) inhibited the differentiation of the SAM and caused the development of a determinate inflorescence. Nevertheless, the positive T1 A. thaliana plants grew many buds, showing an indeterminate phenotype (Figure 4c,d). These results showed that BnA10.TFL1 has similar function to TFL1. 


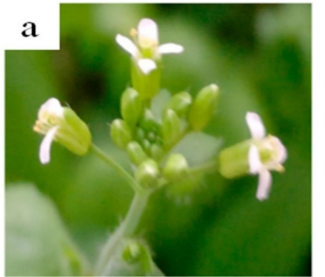

WT

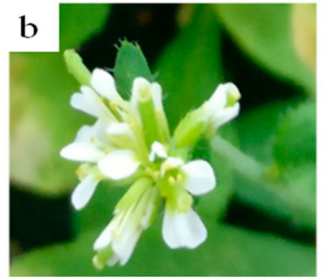

tfl $1-2$

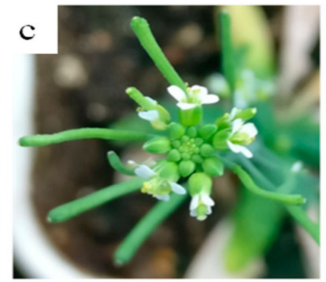

PBNA10.TFL 1:BnA1 O.TFL1-1T 1

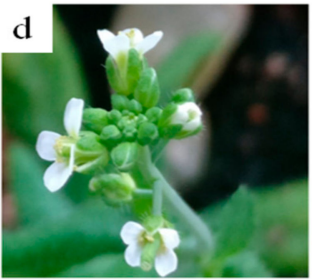

PBNA10.TFL1:BnA1 O.TFL1-2T1

Figure 4. Architecture of terminal racemes in transgenic A. thaliana. (a) Wild-type A. thaliana (WT) shows an indeterminate phenotype. (b) The tfl1-2 mutant has a determinate phenotype with terminal flowers. (c) T1 A. thaliana transgenic plants transformed with $p B n A 10 . T F L 1: B n A 10 . T F L 1-1 T 1$ returned to an indeterminate phenotype. (d) T1 A. thaliana transgenic plants transformed with $p B n A 10 . T F L 1: B n A 10$. TFL1-2T1 also became indeterminate.

\subsection{BnA10.TFL1 Rescues the Determinate Inflorescence Trait in B. napus}

In B. napus, to determine whether the BnA10.TFL1 controls the gene of the inflorescence trait, we transformed the complementation plasmid PBnA10.TFL1:BnA10.TFL1 sequence into the hypocotyl of the determinate line 571 (Figure 5 b) by the Agrobacterium-tumefaciens-mediated method. The transgenic plants containing the $p B n A 10 . T F L 1: B n A 10 . T F L 1$ construct produced numerous buds (Figure $5 \mathrm{c}, \mathrm{d}$ ). These positive plants exhibited phenotypic reversions parallel to the indeterminate inflorescence architecture (Figure 5a). Then, the SAMs of the positive B. napus plants were examined at the budding stage to analyze the expression levels of the gene. The relative expression of BnA10.TFL1 in the indeterminate line 2014 was significantly up-regulated compared with that in the determinate line 571 (Figure 5e). In addition, BnA10.TFL1 showed significantly higher expression in the positive plants than in the determinate line 571 (Figure 5e). Thus, high expression of BnA10.TFL1 was associated with the indeterminate inflorescence growth habit. In conclusion, BnA10.tfl1 is a gene controlling determinate inflorescences in B. napus.

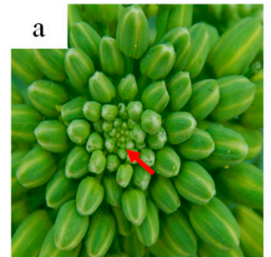

2014-Indeterminate

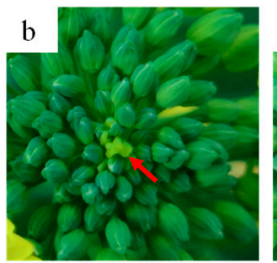

571- Determinate

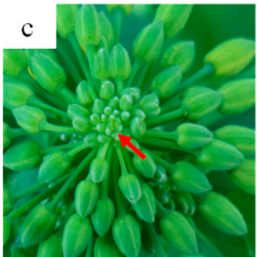

pBnA10.TFL1:BnA10.T FL1-1

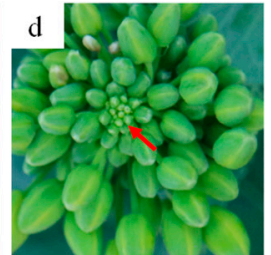

pBNA10.TFL1:BnA10.T FL1-2

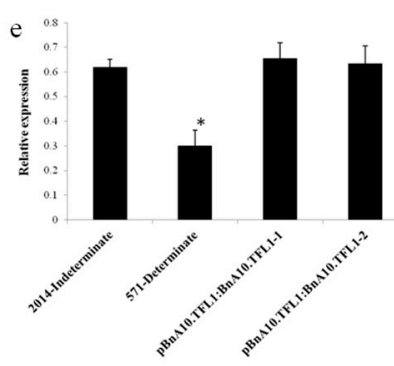

Figure 5. Architecture and BnA10.TFL1 expression in the inflorescence apex of transgenic positive plants in B. napus. (a) The 2014 line is naturally indeterminate. (b) The 571 line has a determinate inflorescence and abnormal floral organs. (c,d) B. napus transgenic plants positive for PBnA10.TFL1:BnA10.TFL1-1 and pBnA10.TFL1:BnA10.TFL1-2 show indeterminate phenotypes. (e) BnA10.TFL1 expression in transgenic positive plant inflorescence apexes in B. napus. Actin7 was used as the reference gene; the error bars show the standard errors from three biological replicates. ${ }^{*}$ indicates significant differences at $p<0.05$. The arrows indicate the inflorescence apexes.

\subsection{Subcellular Localization of the BnA10.TFL1 Protein}

To confirm the subcellular localization of the BnA10.TFL1 protein in B. napus, the fusion plasmid BnA10.TFL1-GFP was transiently expressed in the leaves of tobacco ( $N$. benthamiana). The BnA10.TFL1-GFP protein signals were localized to the nucleus, cytoplasm, and plasma membrane (Figure 6). This provides a further basis for research into the molecular function of BnA10.TFL1. 


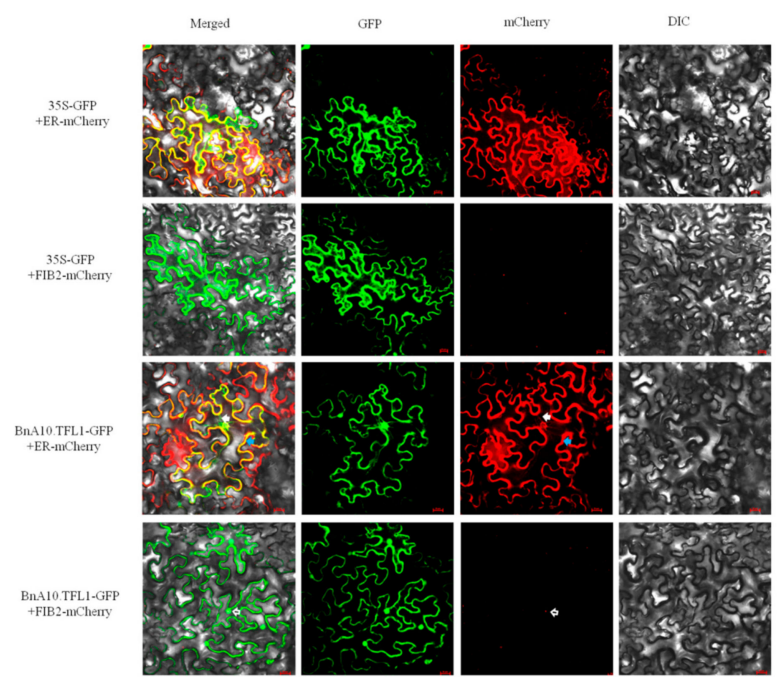

Figure 6. The subcellular location of the BnA10.TFL1 protein in Nicotiana benthamiana epidermal cells. The ER-mCherry was used as a marker for ER-rb CD-960, FIB2-mCherry was used as a nuclear marker, 35S-GFP + ER-mCherry and 35S-GFP + FIB2-mCherry were used as control; the hollow white arrow refers to the nuclear, the solid white arrow refers to the filamentous substance in the cytoplasm, the solid blue arrow refers to plasma membrane; scale bar $=20 \mu \mathrm{m}$.

\section{Discussion}

Plant growth habits (in particular, indeterminate or determinate inflorescences) have an important effect on the architecture and yield of plants such as oilseed [3,6,32], soybean [33,34], and tomato [35]. The development of the SAM determines the plant growth habit; the SAM is located at the top of the stem of the plant and is mainly composed of cells with unlimited growth capacity [36-38]. These SAM cells produce leaf buds, axillary buds, and flower buds that are closely related to the establishment of plant morphology and the economically important harvestable organs [39-41]. The determinate inflorescence trait was caused by development and differentiation of apex SAM $[4,5,14]$. In B. napus, the candidate gene for the inflorescence trait was identified [3]. In this study, the BnA10.TFL1 was differentially more expressed in the inflorescence apex than in other tissues (Figure 2). Therefore, further research on the function of the BnA10.TFL1 gene is necessary for oilseed breeding.

In this study, we observed the morphological characteristics of determinate and indeterminate inflorescences at different growth stages of the SAM in B. napus. The results showed that the SAMs of determinate inflorescences (Figure $1 \mathrm{~d}, \mathrm{e}, \mathrm{j}, \mathrm{k}$ ) were structurally similar to those of indeterminate inflorescences (Figure 1a,b,g,h) at the vegetative meristem (two leaves) and early inflorescence meristem stages (four leaves). However, the SAM of determinate inflorescences (Figure 1f,l) showed differences compared to indeterminate (Figure 1c,i) during the budding period (eight leaves). The apex of the determinate SAM (Figure 1f,l) showed an organ (similar to the shape of a floral organ) which may cause determinate growth, in contrast to the SAM with unlimited differentiation capacity seen in the indeterminate plants (Figure 1c,i). In other reports, the determinate SAMs were observed to form a terminal flower in the $A$. thaliana $t f l 1$ mutant, halting SAM differentiation $[4,8]$. Meanwhile, the centroradialis mutant apex also developed a flower, terminating the normally indeterminate inflorescences in Antirrhinum [38]. In Sesamum indicum L., the shoot apex of determinate inflorescences showed a flower cluster [42]. Although the phenotypes of determinate inflorescences in these plants were different, they shared a common feature: the apex SAM continued to differentiate into new floral tissue, resulting in indeterminate plants becoming determinate.

$\mathrm{Li}$ et al. [3] reported that a candidate gene controlling plant growth habits, found on B. napus chromosome A10, was homologous to A. thaliana TFL1. In our research, the alleles BnA10.TFL1 and $B n A 10 . t f l$ were isolated from indeterminate and determinate plants, respectively, by PCR. The BnA10.TFL1/BnA10.tfl had a $537 \mathrm{bp}$ ORF (open reading frame) which included four exons 
and three introns. The amino acid sequences of the two alleles encoded a protein of 178 amino acids and contained a conserved PEBP domain (Figure 3a,b). The phylogenetic analysis showed that the BnA10.TFL1 and AtTFL1 proteins are highly homologous with $85 \%$ identities (Figure 3b,c). Cloned TFL1 in other plants, such as in rice [43], soybean [44], apple [45], dogwood [46], and Jatropha curcas [47], showed similar results. These studies indicate that BnA10.TFL1 may share a similar function with TFL1.

TFL1 has been reported to not only control inflorescence meristem identity but to also impact inflorescence architecture and the formation of the reproductive phase at the SAM [48,49]. The functions of TFL1 homologous were studied in some plants. In Vigna radiata, Li and Ding et al. found that the $\operatorname{VrDet} 1$ gene was transformed into the $A$. thaliana $\mathrm{t} f l 1$ mutant, the progeny transgenic plants showed indeterminate traits similar to wild-type $A$. thaliana, and the expression level of $\operatorname{VrDet} 1$ at the SAMs of indeterminate plants was higher than that in determinate plants [10]. In Punica granatum L., PgTFL1 was transformed into the tfl1-14 mutant, and the determinate trait restored growth to an indeterminate plant [50]. In this study, a full-length fragment of genomic DNA containing BnA10.TFL1 was cloned from indeterminate plants and transformed into the determinate $t f l 1-2$ mutant (Figure $4 \mathrm{~b}$ ). The positive transgenic plants (Figure $4 \mathrm{c}, \mathrm{d}$ ) were restored to indeterminate inflorescences similar to wild-type A. thaliana (Figure 4a). The complementation plasmid pBnA10.TFL1:BnA10.TFL1 was also introduced into the determinate $B$. napus line 571 (Figure 5 b), and the results showed that the abnormal organ at the apex of the determinate SAM was absent, confirming the restoration of the indeterminate phenotype (Figure 5c,d). Additionally, the expression of BnA10.TFL1 was also up-regulated in the indeterminate line 2014 and in the positive transgenic plants compared with in the determinate line 571 at the budding stage (Figure 5e). Thus, BnA10.TFL1 may have similar functions to A. thaliana TFL1, which controls inflorescence architecture, as it restored determinate inflorescences in an A. thaliana tfl1 mutant that has a terminal flower. Importantly, it also restored determinate inflorescences in the B. napus line 571 that showed a floran organ at the SAM. The low expression of TFL1 gene (AT5G03840) in A. thaliana was caused by the single nucleotide substitution $\mathrm{G}$ to $\mathrm{A}$ at $3^{\prime}$-end of the second exon. In cucumber, what causes (mutation and deletion) to cause down-regulation of the CSTFL1 is unclear [20]. In this study, whether the amino acid, promoter and others changed between indeterminate and determinate may cause binding to downstream genes and expression differences that remains unknown. This will be subject to further study. Previous studies showed that TFL1 is expressed mainly in the SAM, and mobile TFL1 may play a role in vesicle trafficking [48,51]. Hanano and Goto [52] found that the TFL1 protein is localized to the nucleus and cytoplasm. However, CmTFL1c from Chrysanthemum morifolium also belongs to the nucleus and plasma membrane [16]. In this study, BnA10.TFL1 protein was found to localize to the nucleus, cytoplasm, and plasma membrane (Figure 6). Therefore, our findings indicate that BnA10.tfl1 acts an important gene controlling determinate inflorescences in B. napus.

\section{Conclusions}

In summary, the present study revealed mainly that the function of BnA10.tfl1 controls determinate inflorescences in B. napus. First of all, we analyzed the morphological development of SAMs and gene expression by scanning electron microscopy, paraffin sectioning techniques, and qRT-PCR. The results showed that BnA10.tfl1 may be specifically expressed in the shoot apex, and down-regulation of its expression was observed in determinate inflorescence plants. Phylogenetic analysis showed that the BnA10.TFL1 protein has high similarity with $A$. thaliana TFL1. Further, when the BnA10.TFL1 gene from an indeterminate inflorescence plant was transferred to determinate inflorescence $A$. thaliana and B. napus, the determinate traits were turned to indeterminate. These results indicate that BnA10.tfl may control the determinate inflorescence trait. All these results taken together, this study may provide new insights into the molecular mechanism of and oilseed breeding for determinate inflorescences.

Supplementary Materials: The following are available online at http://www.mdpi.com/2073-4395/9/11/722/s1, Table S1: The primer sequences used in this study, Figure S1: The 3816 bp genomic fragment incorporating the 
BnA10.TFL1 gene. The green background shows the $1860 \mathrm{bp}$ of the gene upstream, the grey background shows the $1066 \mathrm{bp}$ of the gene coding region, and the yellow background shows the $890 \mathrm{bp}$ of the gene downstream.

Author Contributions: D.D. conceived and designed the research. Y.J., K.L., H.L. and L.Z. performed the experiments and analyzed the data. Y.J. and K.L. wrote and modified the article. All authors read and approved the manuscript.

Funding: This work was supported by the National Key Research and Development Plan of China (2016YFD0101300), the National System of Technology of the Rapeseed Industry (CARS-12), the Key Laboratory of Spring Rape Genetic Improvement of Qinghai Province (2017-ZJ-Y09), and the Natural Science Fund Project of Qinghai Province (2019-ZJ-972Q).

Acknowledgments: We are grateful to Lu Xiao, Liang Xu, Huazhong Agricultural University, and the Oil Crop Research Institute of the Chinese Academy of Agricultural Sciences for their help in purchasing A. thaliana mutants.

Conflicts of Interest: The authors declare no conflict of interest.

\section{References}

1. Prakash, S.; Wu, X.M.; Bhat, S.R. History, Evolution, and Domestication of Brassica Crops. Plant Breed. Rev. 2011, 35, 19-84. [CrossRef]

2. Chalhoub, B.; Denoeud, F.; Liu, S.; Parkin, I.A.; Tang, H.; Wang, X.; Chiquet, J.; Belcram, H.; Tong, C.; Samans, B.; et al. Plant genetics. Early allopolyploid evolution in the post-Neolithic Brassica napus oilseed genome. Science 2014, 345, 950-953. [CrossRef] [PubMed]

3. Li, K.; Yao, Y.; Xiao, L.; Zhao, Z.; Guo, S.; Fu, Z.; Du, D. Fine mapping of the Brassica napus Bnsdt1 gene associated with determinate growth habit. Theor. Appl. Genet. 2018, 131, 193-208. [CrossRef] [PubMed]

4. Alvarez, J.P.; Guli, C.L.; Yu, X.H.; Smyth, D.R. Terminal flower: A gene affecting inflorescence development in Arabidopsis thaliana. Plant J. 1992, 2, 103-116. [CrossRef]

5. Conti, L.; Bradley, D. TERMINAL FLOWER1 is a mobile signal controlling Arabidopsis architecture. Plant Cell 2007, 19, 767-778. [CrossRef] [PubMed]

6. Kaur, H.; Banga, S.S. Discovery and mapping of Brassica juncea Sdt 1 gene associated with determinate plant growth habit. Theor. Appl. Genet. 2015, 128, 235-245. [CrossRef] [PubMed]

7. Ravelombola, W.; Qin, J.; Shi, A.N.; Weng, Y.J.; Bhattarai, G.; Dong, L.D.; Morris, J.B. A SNP-based association analysis for plant growth habit in worldwide cowpea (Vigna unguiculata (L.) Walp) Germplasm. Euphytica 2017, 213, 284. [CrossRef]

8. Shannon, S.; Meeks-Wagner, D.R. Genetic Interactions That Regulate Inflorescence Development in Arabidopsis. Plant Cell 1993, 5, 639-655. [CrossRef]

9. Bradley, D.; Ratcliffe, O.; Vincent, C.; Carpenter, R.; Coen, E. Inflorescence commitment and architecture in Arabidopsis. Science 1997, 275, 80-83. [CrossRef]

10. Li, S.; Ding, Y.; Zhang, D.; Wang, X.; Tang, X.; Dai, D.; Jin, H.; Lee, S.H.; Cai, C.; Ma, J. Parallel domestication with a broad mutational spectrum of determinate stem growth habit in leguminous crops. Plant J. 2018, 96, 761-771. [CrossRef]

11. Olsen, K.M.; Womack, A.; Garrett, A.R.; Suddith, J.I.; Purugganan, M.D. Contrasting evolutionary forces in the Arabidopsis thaliana floral developmental pathway. Genetics 2002, 160, 1641-1650. [PubMed]

12. Ahn, J.H.; Miller, D.; Winter, V.J.; Banfield, M.J.; Lee, J.H.; Yoo, S.Y.; Henz, S.R.; Brady, R.L.; Weigel, D. A divergent external loop confers antagonistic activity on floral regulators FT and TFL1. EMBO J. 2006, 25, 605-614. [CrossRef] [PubMed]

13. Simon, R.; Igeno, M.I.; Coupland, G. Activation of floral meristem identity genes in Arabidopsis. Nature 1996, 384, 59-62. [CrossRef] [PubMed]

14. Perilleux, C.; Bouche, F.; Randoux, M.; Ormanligeza, B. Turning meristems into fortresses. Trends Plant Sci. 2019, 24, 431-442. [CrossRef] [PubMed]

15. Ellul, P.; Angosto, T.; Garciasogo, B.; Garciahurtado, N.; Martintrillo, M.; Salinas, M.; Moreno, V.; Lozano, R.; Martinezzapater, J.M. Expression of Arabidopsis APETALA1 in tomato reduces its vegetative cycle without affecting plant production. Mol. Breed. 2004, 13, 155-163. [CrossRef]

16. Gao, Y.; Gao, Y.; Wu, Z.; Bu, X.; Fan, M.; Zhang, Q. Characterization of TEMINAL FLOWER1 homologs CmTFL1c gene from Chrysanthemum morifolium. Plant Mol. Biol. 2019, 99, 587-601. [CrossRef]

17. Bowman, J.L.; Alvarez, J.; Weigel, D.; Meyerowitz, E.M.; Smyth, D.R. Control of flower development in Arabidopsis thaliana by APETALA1 and interacting genes. Development 1993, 119, 721-743. 
18. Goto, K.; Meyerowitz, E.M. Function and regulation of the Arabidopsis floral homeotic gene PISTILLATA. Genes Dev. 1994, 8, 1548-1560. [CrossRef]

19. Benlloch, R.; Kim, M.C.; Sayou, C.; Thevenon, E.; Parcy, F.; Nilsson, O. Integrating long-day flowering signals: A LEAFY binding site is essential for proper photoperiodic activation of APETALA1. Plant J. 2011, 67, 1094-1102. [CrossRef]

20. Wen, C.; Zhao, W.; Liu, W.; Yang, L.; Wang, Y.; Liu, X.; Xu, Y.; Ren, H.; Guo, Y.; Li, C. CsTFL1 inhibits determinate growth and terminal flower formation through interaction with CsNOT2a in cucumber. Development 2019, 146, dev180166. [CrossRef]

21. Repinski, S.L.; Kwak, M.; Gepts, P. The common bean growth habit gene PvTFL1y is a functional homolog of Arabidopsis TFL1. Theor. Appl. Genet. 2012, 124, 1539-1547. [CrossRef] [PubMed]

22. Fulton, T.M.; Chunwongse, J.; Tanksley, S.D. Microprep protocol for extraction of DNA from tomato and other herbaceous plants. Plant Mol. Biol. Rep. 1995, 13, 207-209. [CrossRef]

23. Sun, Q.; Xue, J.; Lin, L.; Liu, D.; Wu, J.; Jiang, J.; Wang, Y. Overexpression of Soybean Transcription Factors GmDof4 and GmDof11 Significantly Increase the Oleic Acid Content in Seed of Brassica napus L. Agronomy 2018, 8, 222. [CrossRef]

24. Chen, C.; Xiao, L.; Li, X.; Du, D. Comparative Mapping Combined With Map-Based Cloning of the Brassica juncea Genome Reveals a Candidate Gene for Multilocular Rapeseed. Front. Plant Sci. 2018, 9, 1744. [CrossRef] [PubMed]

25. Teng, C.; Du, D.; Xiao, L.; Yu, Q.; Shang, G.; Zhao, Z. Mapping and Identifying a Candidate Gene (Bnmfs) for Female-Male Sterility through Whole-Genome Resequencing and RNA-Seq in Rapeseed (Brassica napus L.). Front. Plant Sci. 2017, 8, 2086. [CrossRef] [PubMed]

26. Xiao, L.; Li, X.; Liu, F.; Zhao, Z.; Xu, L.; Chen, C.; Wang, Y.; Shang, G.; Du, D. Mutations in the CDS and promoter of BjuA07.CLV1 cause a multilocular trait in Brassica juncea. Sci. Rep. 2018, 8, 5339. [CrossRef] [PubMed]

27. Bent, A. Arabidopsis thaliana floral dip transformation method. Methods Mol. Biol. 2006, 343, 87-103. [CrossRef]

28. Zhou, Y.; Wang, H.; Gilmer, S.; Whitwill, S.; Keller, W.; Fowke, L.C. Control of petal and pollen development by the plant cyclin-dependent kinase inhibitor ICK1 in transgenic Brassica plants. Planta 2002, 215, 248-257. [CrossRef]

29. Sang, J.; Wang, Z.; Li, M.; Cao, J.; Niu, G.; Xia, L.; Zou, D.; Wang, F.; Xu, X.; Han, X.; et al. ICG: A wiki-driven knowledgebase of internal control genes for RT-qPCR normalization. Nucleic Acids Res. 2018, 46, D121-D126. [CrossRef]

30. Livak, K.J.; Schmittgen, T.D. Analysis of relative gene expression data using real-time quantitative PCR and the 2(-Delta Delta C(T)) Method. Methods 2001, 25, 402-408. [CrossRef]

31. Nelson, B.K.; Cai, X.; Nebenfuhr, A. A multicolored set of in vivo organelle markers for co-localization studies in Arabidopsis and other plants. Plant J. 2007, 51, 1126-1136. [CrossRef] [PubMed]

32. Kaur, H.; Gupta, S.; Kumar, N.; Akhatar, J.; Banga, S.S. Progression of molecular and phenotypic diversification in resynthesized Brassica juncea (L) gene pool with determinate inflorescence. Euphytica 2014, 199, 325-338. [CrossRef]

33. Ablett, G.R.; Beversdorf, W.D.; Dirks, V.A. Row Width and Seeding Rate Performance of Indeterminate, Semideterminate, and Determinate Soybean. J. Prod. Agric. 1991, 4, 391-395. [CrossRef]

34. Hartman, D.J.; Slivka, A.; Giusto, D.A.; Krasinskas, A.M. Tissue yield and diagnostic efficacy of fluoroscopic and cholangioscopic techniques to assess indeterminate biliary strictures. Clin. Gastroenterol. Hepatol. 2012, 10, 1042-1046. [CrossRef]

35. Maboko, M.M.; Du Plooy, C.P. Response of Field-Grown Indeterminate Tomato to Plant Density and Stem Pruning on Yield. Int. J. Veg. Sci. 2018, 24, 612-621. [CrossRef]

36. Barton, M.K.; Poethig, R.S. Formation of the shoot apical meristem in Arabidopsis thaliana: An analysis of development in the wild-type and in the SHOOT MERISTEMLESS mutant. Development 1993, 119, 823-831.

37. Sautter, C.; Leduc, N.; Bilang, R.; Iglesias, V.A.; Gisel, A.; Wen, X.; Potrykus, I. Shoot apical meristems as a target for gene transfer by microballistics. Euphytica 1995, 85, 45-51. [CrossRef]

38. Bradley, D.; Carpenter, R.; Copsey, L.; Vincent, C.; Rothstein, S.; Coen, E. Control of inflorescence architecture in Antirrhinum. Nature 1996, 379, 791-797. [CrossRef] 
39. Long, J.A.; Barton, M.K. The development of apical embryonic pattern in Arabidopsis. Development 1998, $125,3027-3035$.

40. Dong, H.Z.; Zhang, D.M.; Tang, W.; Li, W.J.; Li, Z. Effects of planting system, plant density and flower removal on yield and quality of hybrid seed in cotton. Field Crops Res. 2005, 93, 74-84. [CrossRef]

41. Truskina, J.; Vernoux, T. The growth of a stable stationary structure: Coordinating cell behavior and patterning at the shoot apical meristem. Curr. Opin. Plant Biol. 2018, 41, 83-88. [CrossRef] [PubMed]

42. Zhang, H.; Miao, H.; Li, C.; Wei, L.; Duan, Y.; Ma, Q.; Kong, J.; Xu, F.; Chang, S. Ultra-dense SNP genetic map construction and identification of SiDt gene controlling the determinate growth habit in Sesamum indicum L. Sci. Rep. 2016, 6, 31556. [CrossRef] [PubMed]

43. Nakagawa, M.; Shimamoto, K.; Kyozuka, J. Overexpression of RCN1 and RCN2, rice TERMINAL FLOWER1/CENTRORADIALIS homologs, confers delay of phase transition and altered panicle morphology in rice. Plant J. 2002, 29, 743-750. [CrossRef] [PubMed]

44. Tian, Z.; Wang, X.; Lee, R.; Li, Y.; Specht, J.E.; Nelson, R.L.; McClean, P.E.; Qiu, L.; Ma, J. Artificial selection for determinate growth habit in soybean. Proc. Natl. Acad. Sci. USA 2010, 107, 8563-8568. [CrossRef]

45. Flachowsky, H.; Szankowski, I.; Waidmann, S.; Peil, A.; Trankner, C.; Hanke, M.V. The MdTFL1 gene of apple (Malus $x$ domestica Borkh.) reduces vegetative growth and generation time. Tree Physiol. 2012, 32, 1288-1301. [CrossRef]

46. Liu, X.; Zhang, J.; Abuahmad, A.; Franks, R.G.; Xie, D.Y.; Xiang, Q.Y. Analysis of two TFL1 homologs of dogwood species (Cornus L.) indicates functional conservation in control of transition to flowering. Planta 2016, 243, 1129-1141. [CrossRef]

47. Li, C.; Fu, Q.; Niu, L.; Luo, L.; Chen, J.; Xu, Z.F. Three TFL1 homologues regulate floral initiation in the biofuel plant Jatropha curcas. Sci. Rep. 2017, 7, 43090. [CrossRef]

48. Wickland, D.P.; Hanzawa, Y. The FLOWERING LOCUS T/TERMINAL FLOWER1 Gene Family: Functional Evolution and Molecular Mechanisms. Mol. Plant 2015, 8, 983-997. [CrossRef]

49. Kathryn, K.M.; Lívia, L.C.; Henrik, L.; Renate, M. Phylogenomic Analysis of the PEBP Gene Family from Kalanchoë. Agronomy 2019, 9, 171. [CrossRef]

50. Patil, H.B.; Chaurasia, A.K.; Azeez, A.; Krishna, B.; Subramaniam, V.R.; Sane, A.P.; Sane, P.V. Characterization of two TERMINAL FLOWER1 homologs PgTFL1 and PgCENa from pomegranate (Punica granatum L.). Tree Physiol. 2018, 38, 772-784. [CrossRef]

51. Serrano-Mislata, A.; Fernandez-Nohales, P.; Domenech, M.J.; Hanzawa, Y.; Bradley, D.; Madueno, F. Separate elements of the TERMINAL FLOWER1 cis-regulatory region integrate pathways to control flowering time and shoot meristem identity. Development 2016, 143, 3315-3327. [CrossRef] [PubMed]

52. Hanano, S.; Goto, K. Arabidopsis TERMINAL FLOWER1 is involved in the regulation of flowering time and inflorescence development through transcriptional repression. Plant Cell 2011, 23, 3172-3184. [CrossRef] [PubMed]

(C) 2019 by the authors. Licensee MDPI, Basel, Switzerland. This article is an open access article distributed under the terms and conditions of the Creative Commons Attribution (CC BY) license (http://creativecommons.org/licenses/by/4.0/). 